\title{
Defect-mode-like transmission and localization of light in photonic crystals without defects
}

\author{
A. E. Serebryannikov* \\ Technische Universitaet Hamburg-Harburg, E-3, D-21071 Hamburg, Germany \\ Ekmel Ozbay \\ Nanotechnology Research Center, Department of Physics, Department of Electrical and Electronics Engineering, \\ Bilkent University, 06800 Ankara, Turkey \\ P. V. Usik \\ Institute of Radio Astronomy, National Academy of Sciences of Ukraine, 61085 Kharkiv, Ukraine
}

(Received 24 February 2010; published 29 October 2010)

\begin{abstract}
It is shown that far- and near-field effects, which are similar to those arising in transmission through finite-thickness photonic crystals (PCs) with structural defects, can be obtained in the conventional defect-free dielectric square-lattice PCs. Multiple narrow peaks of total transmission can appear within a frequency range, where the noncircular isofrequency dispersion contours of one type are quickly transformed for a higher-order Floquet-Bloch wave to the noncircular contours of the other type, leading to a frequency-domain passband being very narrow within a certain range of variation of the angles of incidence. In this regime, the mirror reflectance of the equivalent Fabry-Pérot resonator takes rather large values, which correspond to large values of $Q$ factor and group index of refraction, strong field localization, and good isolation of the transmission peaks from each other. In some examples presented, $Q$ factor exceeds $10^{4}$.
\end{abstract}

DOI: 10.1103/PhysRevB.82.165131

PACS number(s): 42.70.Qs, 42.25.Bs, 78.20.Ci, 42.79.Ci

\section{INTRODUCTION}

Localization of electromagnetic field at structural defects, i.e., the appearance of defect modes, is one of the most interesting features demonstrated by one-, two-, and threedimensional photonic crystals (PCs). Various defect-mode related transmission and waveguide regimes have been in the focus of interest for more than one decade. They were successfully utilized in filters, waveguides, resonators, splitters, mode converters, (de-) multiplexers, tapers, cavities, couplers, detectors, low-threshold lasers, and buffers, e.g., see Refs. 1-13.

Defect modes manifest themselves in the transmission regime in finite-thickness pieces of PCs due to the appearance of narrow transmission peaks. In the dielectric PCs with multiple narrow coupled defects and those with a single wide defect, multiple transmission peaks can appear within a stop band of the corresponding defect-free PC. They are used, for example, in multiple-wavelength frequency filters and multiband spatial filters. ${ }^{13-15}$ Unusual transmission features can be realized when defects are made of a left-handed material, for example, enabling multiple-wavelength omnidirectional filters. $^{16}$

The defect-mode originated transmittance can be interpreted in terms of an equivalent Fabry-Pérot resonator with either planar mirrors placed at virtual interfaces of a PC (in the both cases of multiple narrow defects and a single wide defect), or volumetric mirrors representing pieces of a regular PC, which delimit a wide defect working as a resonator. ${ }^{13}$ In a lossless case, defect modes lead to the total-transmission peaks. Being weakly coupled to each other, these modes can show high $Q$ values, while the peaks are well isolated from each other. In other words, the defect modes can provide one with large values of reflectance of an equivalent planar mirror $\hat{R}$. The larger the $\hat{R}$, the larger $Q$ factor and the better the isolation are. In planar homogeneous slabs, either very high or very low permittivity values are required for obtaining of sufficiently large values of $\hat{R}$. Because of this, the use of PCs, which are made of conventional dielectrics and show structural defects, is quite reasonable.

A way to localize electromagnetic waves without defects in finite-thickness slabs of planar topology is also known, which requires utilization of a negative-index material. ${ }^{17}$ On the other hand, a rich variety of dispersion types, which are achievable in PCs without defects, enables obtaining of the transmission effects like negative refraction without left handedness, highly directive transmission, and bandpass spatial filtering. ${ }^{18-20}$ Besides, a rather wide range of variation in $\hat{R}$ is expected to be obtainable. Indeed, a slab of twodimensional PC without defects itself shows a Fabry-Pérot type behavior of transmittance $T$ within regular passbands, which are associated with Floquet-Bloch (FB) waves. ${ }^{21}$ In the existing theoretical and experimental performances of two-dimensional PCs, typical transmission features indicate an increase in $\hat{R}$ at the edges of a regular passband, which is connected, in turn, with reduction in group velocity. However, the $Q$ factor in these performances is often insufficiently large and the $T$ minima are insufficiently deep in order to consider them as competitors of those with defects. It is noteworthy that reduction of group velocity near the edges is known also in one-dimensional defect-free structures like fiber Bragg gratings. ${ }^{12}$ Recently, the dielectric PCs of circular topology have been suggested, where strong localization can appear within a certain regular layer, leading to the sharp peaks in the frequency dependence of the radiation power. ${ }^{22}$

In this paper, we will show that defect-mode-like multiple peaks of total transmission can be obtained in the finitethickness slabs of classical square-lattice dielectric PCs with- 


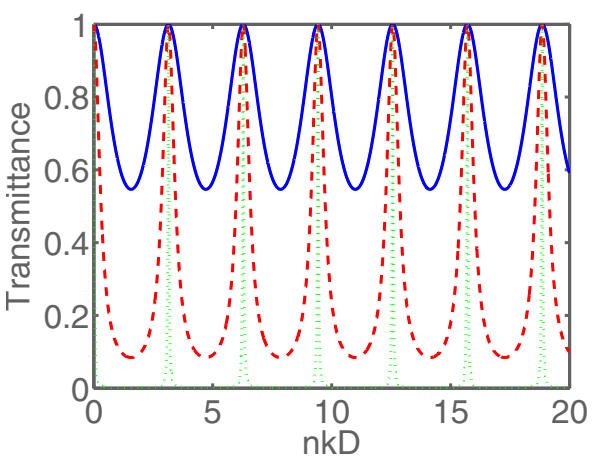

FIG. 1. (Color online) Transmittance as a function of $n k D$ at $\theta$ $=0: \hat{R}=0.15$ - solid blue line, $\hat{R}=0.55$-dashed red line, and $\hat{R}$ $=0.95$ - dotted green line.

out defects, within the narrow (mini-) passbands, which are connected with a quick transformation of the isofrequency dispersion contours (IFCs) that appears while varying frequency. Rather large values of $Q$, good isolation of the peaks of $T$ from each other, and strong field localization can be obtained, giving an alternative in certain operation regimes to the slabs of noncurvilinear lattice PCs with defects.

Our consideration is based on the assumption, according to which the effect that $\hat{R}$ increases close to a passband edge is reinforced for narrow passbands. The desired effects will be demonstrated in square-lattice PCs in case of $s$-polarized plane incident wave, within, and in the vicinity of the frequency range, where the nonisotropic IFCs localized near the $X$ point are transformed to those localized near the $\Gamma$ and $M$ points. Simulations have been carried out for dispersion and transmission by using CST MICROWAVE STUDIO (Ref. 23) and the coupled integral equations based homemade code, ${ }^{24}$ respectively.

\section{THEORETICAL BACKGROUND}

Transmittance of a Fabry-Pérot resonator filled with a homogeneous dielectric dispersionless medium is given by the Airy formula, ${ }^{25}$

$$
T=(1-\hat{R})^{2} /\left[(1-\hat{R})^{2}+4 \hat{R} \sin ^{2}\left(n k D \cos \theta^{\prime}\right)\right],
$$

where $k=\omega / c$ is the free-space wave number, $\theta^{\prime}$ is the angle of refraction, $D$ and $n$ are the distance and index of refraction of the medium between the mirrors, respectively, and $\hat{R}$ is the reflectance of a mirror. In case of a freely located slab, $\hat{R}$ is given by

$$
\hat{R}=\left[\left(\cos \theta-n \cos \theta^{\prime}\right) /\left(\cos \theta+n \cos \theta^{\prime}\right)\right]^{2},
$$

where $n \cos \theta^{\prime}=\sqrt{n^{2}-\sin ^{2} \theta}$ and $\theta$ is the angle of incidence. Figure 1 shows $T$ vs $n k D$ calculated by using Eq. (1) at normal incidence. It is seen that large values of $\hat{R}$ are required for obtaining well-isolated narrow peaks, which are similar to those arising in the slabs of PC due to the structural defects.

According to Eq. (2), $n=n^{ \pm}$is expressed in terms of $\hat{R}$ as follows:
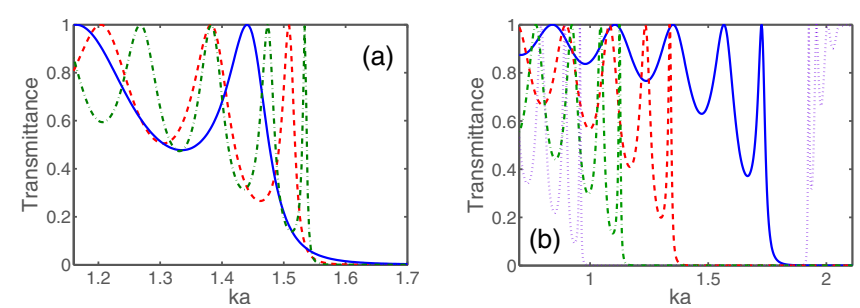

FIG. 2. (Color online) Transmittance vs $k a$ at (a) $d / a=0.4, \varepsilon_{r}$ 11.4, $N=5$-solid blue line, $N=8$-dashed red line, and $N=12$-dash-dotted green line, and (b) $d / a=0.4, \quad N=8$, $\varepsilon_{r}=8.2$-solid blue line, $\varepsilon_{r}=15$-dashed red line, $\varepsilon_{r}=22$-dash-dotted green line, and $\varepsilon_{r}=31$-dotted violet line; $\theta$ $=0$.

$$
\left(n^{ \pm}\right)^{2}=\cos ^{2} \theta\left(\frac{1-\hat{R}^{1 / 2}}{1+\hat{R}^{1 / 2}}\right)^{\mp 2}+\sin ^{2} \theta .
$$

For example, $n^{+}=78$ and $n^{-}=1.28 \times 10^{-2}$ at $\hat{R}=0.95$ in Fig. 1 , where $60<Q<400$ and the minimal transmittance between neighboring peaks is $T_{\min } \approx 6.6 \times 10^{-4}$. In this case, $Q>10^{3}$ starting from $n k D=50$. In the classical Fabry-Pérot resonators, which are created by a slab of a homogeneous dispersionless material, the phase index of refraction $(n)$ and group index of refraction $\left(n_{g}\right)$ have the same values. In slabs of the structured materials, $n \neq n_{g}$ in the general case so that the distance between the neighboring transmission peaks is associated with $n_{g}$.

Despite the progress in obtaining slow light in periodic structures with defects (e.g., see Refs. 12 and 26), design of such structures that shows a desired large value of $n_{g}$ can still be a challenging task. At the same time, materials with 0 $<\operatorname{Re} n<1$ either exist in the nature (noble metals) or can be designed (wire media), depending on the frequency range. ${ }^{27}$ However, because of unavoidable significant losses, they do not allow obtaining peaks of $T=1$. Furthermore, if multiple, closely spaced peaks are required, another problem may occur, which is related to the distance achievable between the neighboring peaks. On the other hand, the $Q$ factor in Fig. 1 is not sufficiently large even at $\hat{R}=0.95$. These and other difficulties are expected to be partially overcame by exploiting the effect of increase of $\hat{R}$ that can appear at a passband edge of a PC, which is made of a conventional low-loss dielectric with a relatively small $n$.

A typical example of $T$ vs $k a$ ( $a$ is the lattice constant) is shown in Fig. 2 at the edge of the lowest passband of a square-lattice dielectric PC for several values of the number of the rod layers $N$ and the relative permittivity of the rods $\varepsilon_{r}$, at an intermediate rod-diameter-to-lattice-constant ratio, $d / a$. The alternating minima and maxima of $T$ indicate a Fabry-Pérot type behavior. In the considered case, the IFCs are located near $\Gamma$ point and, except for the vicinity of the band edge, show the (near-) circular shape, i.e., that is rather close to the IFC shape for a homogeneous isotropic medium.

Figure 2(a) demonstrates the effect of $N$. It is seen that increase in $N$ enables approaching the first near-edge maximum to the passband edge so that it becomes narrower and 
better isolated from its neighbor. This corresponds, in fact, to the increase in $\hat{R}$ for an equivalent Fabry-Pérot resonator. The observed difference in the peak number, which depends on $N$, is also consistent with the theory of the classical Fabry-Pérot resonators. The difference between the effective index of refraction, $n_{\text {eff }}$, which is obtained from the dispersion results, and the equivalent index of refraction obtained from the transmission results, $n_{T}$, is a well known feature. The latter is mainly associated with $n_{g}$ but can also be affected by the peculiarities of treating virtual interfaces of the PC. Dispersion results for Fig. 2(a) (not shown) indicate that $n_{\text {eff }}$ is increased while approaching a passband edge. However, the observed characteristics of the near-edge minimum and maximum of $T$ correspond to $n_{T}>n_{\text {eff }}$.

Figure 2(b) demonstrates the effect of $\varepsilon_{r}$. The basic features observed in Fig. 2(a) remain here. Increase in $\varepsilon_{r}$ leads to that the first near-edge peak of $T$ is better isolated from the neighboring peak. For higher PC passbands, a variation in $\varepsilon_{r}$ can be used for improving the isolation, although an increase in $\varepsilon_{r}$ does not necessarily lead to a better result.

As follows from Eq. (1), the increase in $\theta$ can also result in narrowing and improving isolation of the near-edge peak of $T$. This remains true when the IFCs are located around $M$ point so that $T=0$ at $0<\theta<\theta_{0}$ and $T>0$ at $\theta>\theta_{0}$, as occurs, for example, for the parameters of Fig. 2(a) at $k a>1.55$, at least for $N \geqslant 8$. Giving the guide lines for obtaining a narrow near-edge peak of $T$, the above discussed results do not present such peaks that would be competitive in terms of $Q$ factor and isolation with those achievable in the defect-mode regime. Based on the obtained results (both shown and not shown), we might expect that $\hat{R}$ can rise to the required level if a PC passband is dramatically narrowed.

\section{RESULTS AND DISCUSSION}

\section{A. Existence of minipassbands}

Various IFC shapes and combinations of the group $\left(\mathbf{v}_{g}\right)$ and phase $\left(\mathbf{v}_{\mathrm{ph}}\right)$ velocities are known for higher-order passbands of two-dimensional PCs, which are associated, in particular, with negative refraction, ultrarefraction, and various numbers of the refracted beams, e.g., see Refs. 18, 28, and 29. Among them, there are also those corresponding to the desired narrow passbands. Figure 3 shows an example of a quick transformation of the IFCs for the second lowest PC passband, for which the strong narrowing is possible at intermediate $\theta$.

At the lower boundary of the considered $k a$ range $(k a$ $=2.83$ ), the IFCs are located around $X$ point. Then, they are substantially expanded while keeping nearly the same shape, at least up to $k a=2.99$. Dramatic change in the IFC shape occurs between $k a=2.99$ and $k a=3.03$. As a result, IFCs take a (near-) square shape, being located around $\Gamma$ and $M$ points. A quick change of the shape and width of the IFCs, which determine the coupling scenario, is expected to lead to small group velocity and, hence, to large values of $n_{T}$.

In Fig. 4, three typical cases of coupling of the incident plane wave to a FB wave are demonstrated. Further, we will refer to the cases shown in Figs. 4(a)-4(c), as A, B, and C,

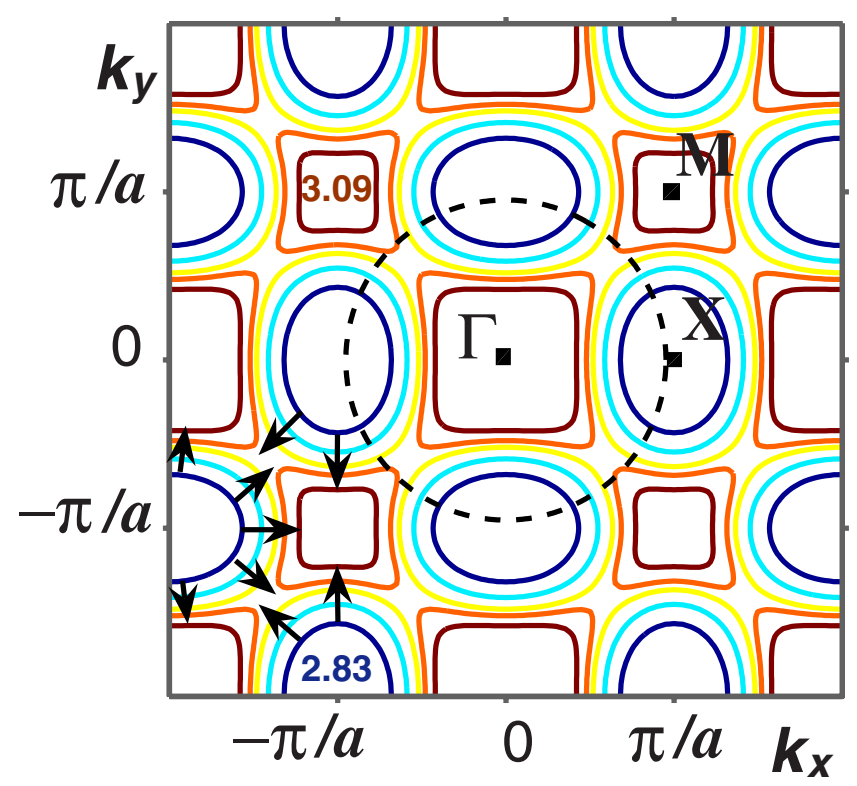

FIG. 3. (Color online) IFCs on the $\left(k_{x}, k_{y}\right)$ plane for PC with $d / a=0.4$ and $\varepsilon_{r}=11.4$ at $k a=2.83$ (dark-blue lines), 2.92 (cyan lines), 2.99 (yellow lines), 3.03 (orange lines), and 3.09 (brown lines); dashed circle-IFC in air at $k a=2.99$; black arrowspossible directions of $\mathbf{v}_{g}$.

respectively, as well as to other similar cases. In the performed analysis of coupling, standard rules and assumptions were utilized..$^{18,29,30}$ In particular, we use conservation of the wave-vector component being parallel to the PC interfaces, $k_{x}$, and consider only those crossing points of IFCs with the construction lines, for which the energy velocity $\mathbf{v}_{e}=\mathbf{v}_{g}$ is pointed away from the source. Note that the knowledge of IFC of the PC at a fixed $\omega$, fixed band slope which is determined by sign of $\nabla_{\mathbf{k}} \omega \cdot \mathbf{k}^{\mathrm{PC}}$, where $\mathbf{k}^{\mathrm{PC}}$ is the wave vector of the FB wave, and gradient of the IFCs at fixed $k_{x}=k_{x}^{\mathrm{PC}}$ is sufficient for determining all the coupled beams. ${ }^{29}$

In case A, the IFCs are located around $X$ point so that $k_{\min }^{(2)}>k_{\max }^{(1)}$, where $k_{\min }^{(2)}$ and $k_{\max }^{(1)}$ mean the minimal value of the modulus of the parallel component of the wave vector $\left|k_{x}\right|$ for the IFCs near $\left|k_{x}\right|=\pi / a$ and the maximal value of $\left|k_{x}\right|$ for the IFCs near $k_{x}=0$, respectively. Here, there is no coupling in the vicinity $\theta=37.5^{\circ}$, while a single beam in the PC is positively refracted for the IFCs located near $k_{x}=0$, i.e., at small $\theta$, and negatively refracted for the IFCs near $\left|k_{x}\right|$ $=\pi / a$, i.e., at large $\theta$. For both the groups of IFCs, $\mathbf{S} \cdot \mathbf{k}^{\mathrm{PC}}$ $<0$, where $\mathbf{S}$ is the Poynting vector.

In case $\mathrm{B}$, the IFCs are still located near the $X$ point. However, now they become so wide that the two refracted beams simultaneously appear in the vicinity of $\theta=37.5^{\circ}$. In turn, at small and large $\theta$, for example, at $\theta=10^{\circ}$ and $\theta$ $=65^{\circ}$, the same features of coupling (not shown) are observed as in case A. Finally, in case $\mathrm{C}$, we again obtain no coupling in the vicinity of $\theta=37.5^{\circ}$. The only significant difference comparing to case A is that $\mathbf{v}_{g}$ is directed now nearly perpendicular to the PC interfaces, indicating a collimation regime. As a result of the described transformation, the width of the $\omega$-domain passband can be minimized at a proper choice of $\theta$. The minimal width $\Delta k a \approx 0.07$ is achieved at $\theta \approx 37.5^{\circ}$. 

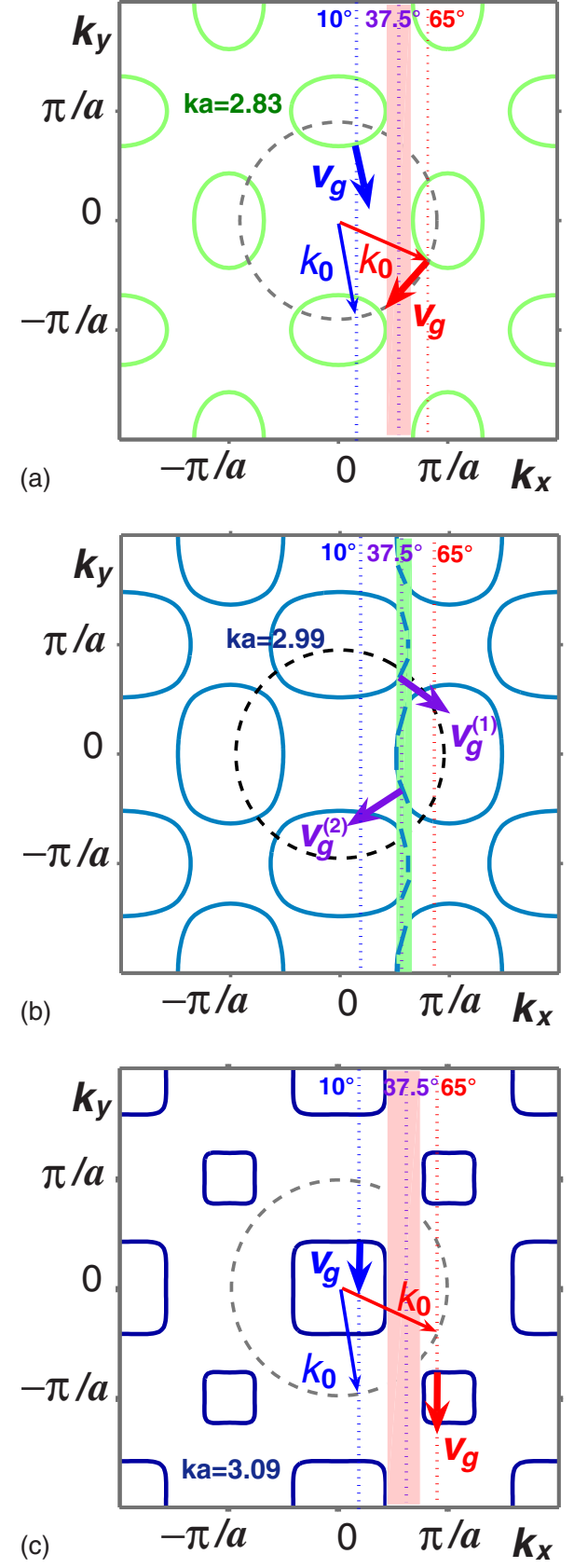

FIG. 4. (Color online) IFCs at (a) $k a=2.83$, (b) 2.99, and (c) 3.09; dashed circles-IFC in air; straight dotted lines-construction lines at $\theta=10^{\circ}, \theta=37.5^{\circ}$, and $\theta=65^{\circ} ; \mathbf{k}=\mathbf{k}_{0}$ (thinner arrows) incident wave vector at $\theta=10^{\circ}$ and $\theta=65^{\circ}$ in plots (a) and (c); thicker arrows-directions of group velocity of the sole refracted beam at $\theta=10^{\circ}$ and $\theta=65^{\circ}$ in plots (a) and (c), and those of the two refracted beams at $\theta=37.5^{\circ}$ in plot (b); rectangles in plots (a) and (c) show the range of variation in $k_{x}$, where no coupling takes place; rectangle in plot (b) shows the $k_{x}$ range with the two refracted beams; the incidence interface is assumed to be parallel to the $k_{x}$ axis and located on the side of positive values of $k_{y}$.

\section{B. Transmission spectra}

Figure 5 presents $T$ as a function of $k a$ for the eight-layer PC with the same $\varepsilon_{r}$ and $d / a$ as in Figs. 3 and 4 at several values of $\theta$, for which the second lowest passband can be
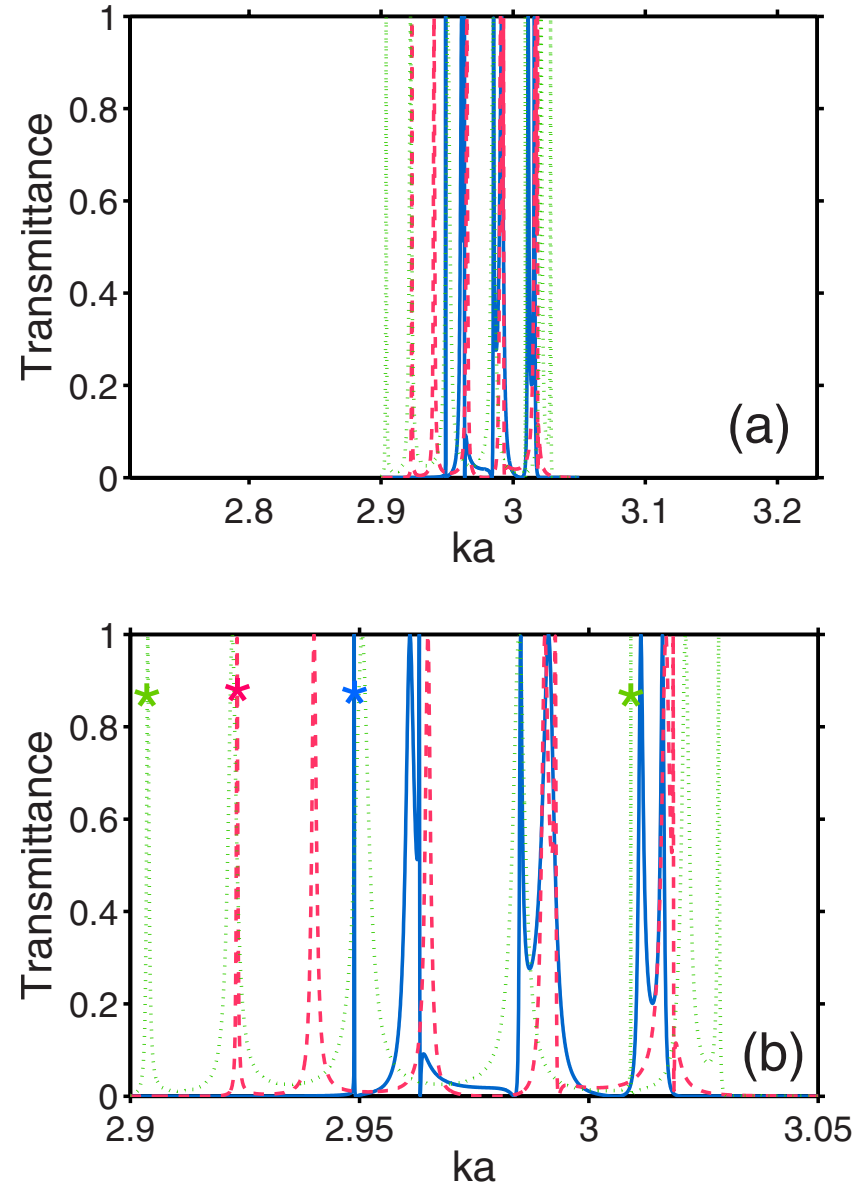

FIG. 5. (Color online) Transmittance vs $k a$ within the minipassband, which is associated with the second lowest FB wave of PC with $d / a=0.4, \varepsilon_{r}=11.4$, and $N=8$ at $\theta=37.5^{\circ}$ (solid blue line), $\theta$ $=40^{\circ}$ (dashed red line), and $\theta=42^{\circ}$ (dotted green line); plot (a)- the passband and the adjacent parts of the first and second stop bands and plot (b) — detailed structure of the passband; some high- $Q$ peaks are denoted by asterisk.

considered as a minipassband. As expected, it consists of the well-isolated multiple narrow peaks of $T=1$. Hence, defectmode-like transmission is obtained without introducing structural defects.

All the peaks of $T=1$ at $k a<2.95$ correspond here to the sole negatively refracted beam, as in case A in Fig. 4(a), for the IFCs located near $\left|k_{x}\right|=\pi / a$. This is also related to the single peaks seen at $2.95<k a<3$. The twin peaks at $k a$ $>2.95$, except for that arising between $k a=3.02$ and $k a$ $=3.03$ at $\theta=42^{\circ}$, correspond to the two simultaneously existing beams with different $\operatorname{sgn}\left(\mathbf{v}_{g} \cdot \mathbf{x}\right)$, as in case B in Fig. 4(b). Here, $\mathbf{x}$ denotes the axis that is parallel to the PC interfaces. It is noteworthy that the opposite edges of the stop bands, which are adjacent to the minipassband, are located at $k a$ $\approx 1.6$ and $k a \approx 3.27$ when $\theta=37.5^{\circ}$.

In line with Eq. (1), where we take $n=n_{T}$, and assuming that $n_{T}$ is not varied between two neighboring peaks of $T, n_{T}$ can be estimated from the location of the peaks. For example, location of the first and second near-edge peaks, which appear in Fig. 5 at $\theta=40^{\circ}$ in the vicinity of $k a=2.93$ is the same as in case of a homogeneous slab with $n \approx 23.4$ and 

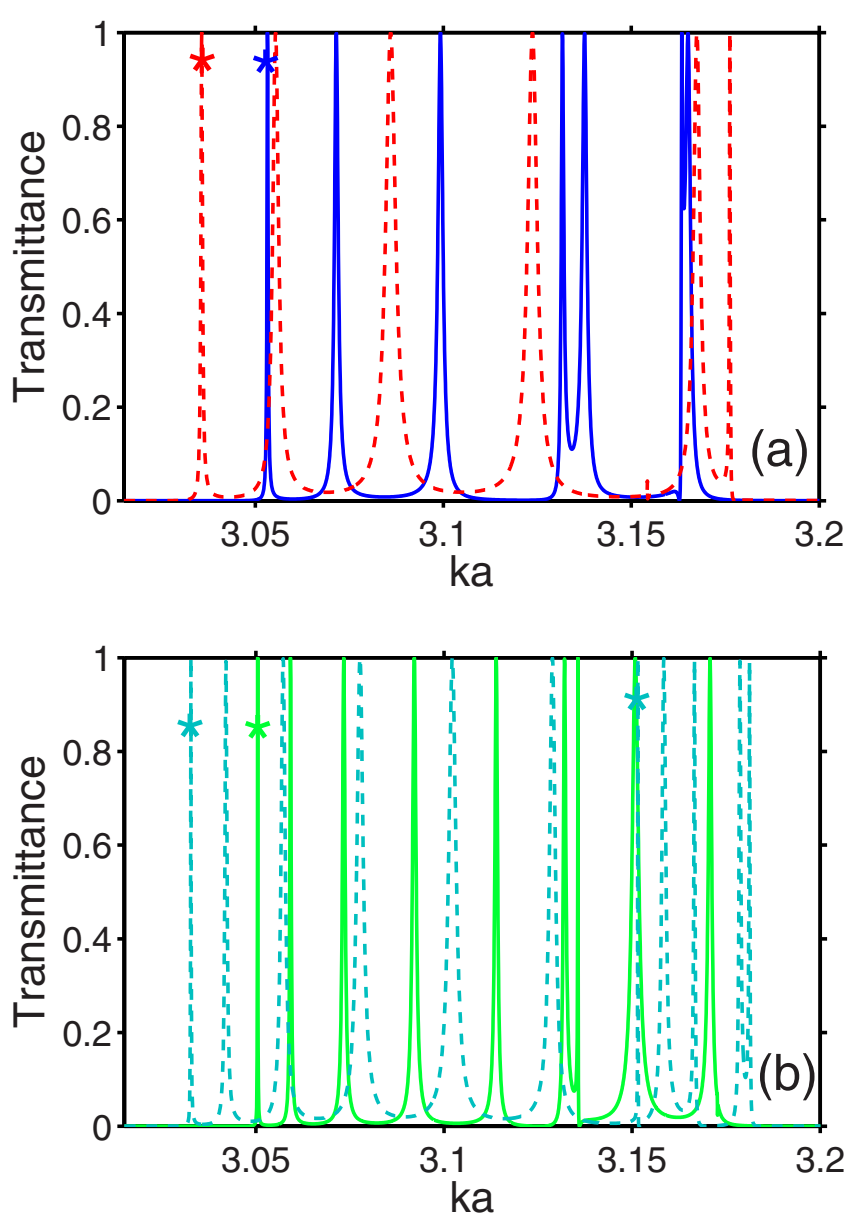

FIG. 6. (Color online) Transmittance vs $k a$ within the minipassband at $d / a=0.4$ and $\varepsilon_{r}=9.61$ : plot (a) $-\theta=38.5^{\circ}$ and $N=8$ (solid blue line), and $\theta=40^{\circ}$ and $N=8$ (dashed red line); plot (b) $-\theta$ $=38.5^{\circ}$ and $N=12$ (solid green line), and $\theta=40^{\circ}$ and $N=12$ (dashed bright-blue line); some high- $Q$ peaks are denoted by asterisk; note the peak densening at increase in $N$.

$D=N a$. Substituting this value of $n$ to Eq. (2), we obtain $\hat{R}$ $\approx 0.877$. On the other hand, the same $T_{\min } \approx 4.6 \times 10^{-3}$, as between the mentioned near-edge peaks, requires $\hat{R} \approx 0.873$. This follows from the comparison with the results for $T$ obtained from Eq. (1) at different $\hat{R}$. Finally, following Eq. (1), $\hat{R}>0.99$ is required in order to obtain the same value of $Q$ $\approx 7.9 \times 10^{3}$ as for the first near-edge peak of $T$, arising at $k a=2.923$. It is worth noting that in our case, the observed location of the peaks is obtained due to a negatively refracted beam (see Figs. 5 and 6) so that a negative value can be assigned to $n_{T}$.

The peaks can be tuned by varying $\theta$, which affects their location and $Q$ factor in similar manner as can be done in the case of defect modes. ${ }^{13}$ The $Q$ values for the peaks denoted in Fig. 5(b) by * are presented in the upper part of Table I. One can see that high- $Q$ peaks can be obtained both at the edge and inside the minipassband. $Q$ factor can further be increased by increasing $N$. At the same time, the features seen in Fig. 5(b) still remain at smaller $N$, e.g., at $N=5$.

The highest $Q$ is often achieved for the single peaks arising in the vicinity of the smaller- $k a$ edge, which correspond
TABLE I. Values of $Q$ for the narrow transmission peaks and the corresponding values of $k a, N$, and $\theta$.

\begin{tabular}{crcc}
\hline \hline$k a$ & $N$ & $\theta$ & $Q$ \\
\hline & & Figure 5(b) & \\
2.904 & 8 & $42^{\circ}$ & $4.8 \times 10^{3}$ \\
2.923 & 8 & $40^{\circ}$ & $7.9 \times 10^{3}$ \\
2.949 & 8 & $37.5^{\circ}$ & $4.2 \times 10^{4}$ \\
3.009 & 8 & $42^{\circ}$ & $4.9 \times 10^{4}$ \\
& & & \\
& & Figure 6 & $1.64 \times 10^{4}$ \\
3.0333 & 12 & $40^{\circ}$ & $5.7 \times 10^{3}$ \\
3.0357 & 8 & $40^{\circ}$ & $2.5 \times 10^{4}$ \\
3.0505 & 12 & $38.5^{\circ}$ & $8.4 \times 10^{3}$ \\
3.0532 & 12 & $38.5^{\circ}$ & $2.8 \times 10^{4}$ \\
3.1515 & $40^{\circ}$ & \\
\hline \hline
\end{tabular}

to the case when a sole coupled beam is negatively refracted. This regime can be interpreted as negative ultrarefraction, since $\theta<\left|\theta^{\prime}\right|$ and $\operatorname{sgn}\left(\mathbf{v}_{g} \cdot \mathbf{x}\right)<0$ while $\operatorname{sgn}\left(\mathbf{k}_{0} \cdot \mathbf{x}\right)>0$ at $\theta$ $>0$. In the limiting case of this regime, $\mathbf{v}_{g} \cdot \mathbf{x} \approx-\left|\mathbf{v}_{g}\right||\mathbf{x}|$ and $\left|k_{x}\right|=k_{\min }^{(2)}$. However, the first near-edge peak is always located at some distance from the band edge. Correspondingly, $\theta^{\prime}$ for this peak can take values from a rather wide range, depending on $N$ and $\theta$.

The discussed effects remain within a wide range of variation in $\varepsilon_{r}, d / a$, and $N$. An example is presented in Fig. 6 for a smaller $\varepsilon_{r}$. As in Fig. 5, the appearance of the minipassband, which comprises the multiple peaks of $T$, is connected with the peculiar quick transformation of the IFCs. As expected, larger $Q$ values are obtained at $N=12$ than at $N=8$. The $Q$ values for the cases denoted by ${ }^{*}$ in Fig. 6 are presented in the lower part of Table I.

\section{Frequency-angle map of transmission}

To better understand the basic features of transmission, it has also been studied at the simultaneous variation in $k a$ and $\theta$. The results are shown in Fig. 7 at the same PC parameters as in Fig. 5. In this case, less fine discretization than in Fig. 5 was used in the coupled integral equation based code in order to reduce the required CPU time to reasonable values. Therefore, locations of the passbands and peaks of $T$ can be slightly different from those in Fig. 5. As compared to a finer discretization, some features can be lost, but the basic ones remain.

The alternating mountains and valleys of $T$ in Fig. 7 indicate the Fabry-Pérot type behavior. Three typical $k a$ ranges can be distinguished, which are denoted by $\mathrm{a}, \mathrm{b}$, and $\mathrm{c}$. They are partially consistent with cases A, B, and C in Fig. 4. The boundary between the $k a$ ranges denoted by a $\left(k_{\min }^{(2)}>k_{\max }^{(1)}\right)$ and $\mathrm{b}\left(k_{\min }^{(2)}<k_{\max }^{(1)}\right)$, which is shown by the left dashed line in Fig. 7. For the both ranges a and b, the IFCs are located near the $X$ point. The boundary between the ranges $\mathrm{b}$ and $\mathrm{c}$ $\left(k_{\min }^{(2)}>k_{\max }^{(1)}\right)$ corresponds to the second case of $k_{\min }^{(2)}=k_{\max }^{(1)}$. Here, the transformation of the IFCs is finished so that they 


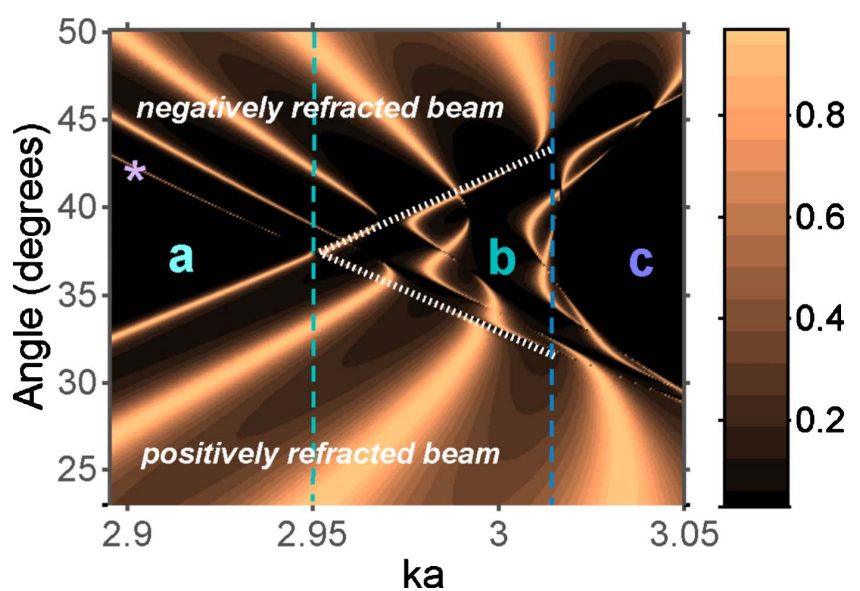

FIG. 7. (Color online) Transmittance on the $(k a, \theta)$ plane for the second lowest FB wave at $d / a=0.4, \varepsilon_{r}=11.4$, and $N=8$; asterisk indicates here the narrow mountain of $T=1$, which corresponds to the peaks denoted by asterisk in Fig. 5(b) at $k a<2.95$.

are located in the range $\mathrm{c}$ around $\Gamma$ and $M$ points. For the range $b$ in Fig. 7 , the regime of two refracted beams occurs inside the range of $\theta=f(k a)$, which is bounded by the dotted white lines (case B in Fig. 4 also belongs to this range). Beyond it, a sole refracted beam contributes to $T$. Due to the peculiar behavior of the mountains of $T$ in the range $b$, the possibility exists to decrease distance between two neighboring peaks at given $\theta$ to a desired value. Hence, the mimicking of $n_{T}$ that is much larger than 23.4 (see Sec. III B) is expected to be obtainable within narrow ranges of $k a$ variation.

Comparing Figs. 5 and 7 and the data from Table I for range a, one can see that the $Q$ factor for the mountain/peak denoted by ${ }^{*}$ varies continuously from $4.8 \times 10^{3}$ at $\theta=42^{\circ}$ to $4.2 \times 10^{4}$ at $\theta=37.5^{\circ}$. According to the obtained results, the width of the passband $\Delta k a \approx 0.13$ is still sufficiently small for obtaining of $Q>4 \times 10^{3}$. Decrease in $\Delta k a$ owing to a proper variation in $\theta$ can result in larger values of $Q$. It is interesting that the near-edge peaks are narrower for the negatively refracted beam, i.e., at larger $\theta$, than for the positively refracted beam, i.e., at smaller $\theta$, while $k a=$ const. Thus, stronger field localization is expected in the former case, although $\mathbf{S} \cdot \mathbf{k}^{\mathrm{PC}}<0$ for both positively and negatively refracted beams.

\section{Field inside PC}

The large values of $Q$-factor like those inferred from Figs. 5 and 6 serve a signature of that the field is strongly localized inside the slab of PC. Let us consider the field distribution for several typical peaks of $T$. Four examples are presented in Fig. 8. In plots (b), (c), (d), and (e), the ratio of the maximal field amplitude in $\mathrm{PC}$ to that of the incident plane wave is nearly equal to $20,70,25$, and 90, respectively. Hence, field can be strongly confined within the defect-free slab of PC in a similar way as within structural defects in a slab of PC. The observed field profiles are symmetric with respect to the PC midplane. However, because of the peculiar field structure inside the slab, these regimes are not the full ana-

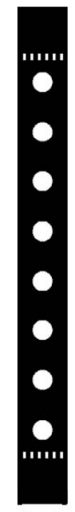

(a) (b)

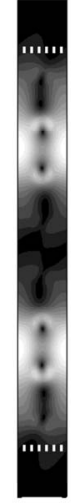

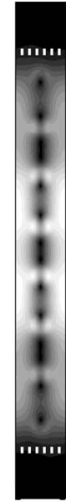

(c)

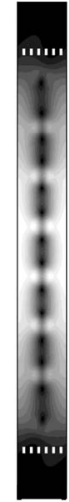

(d)

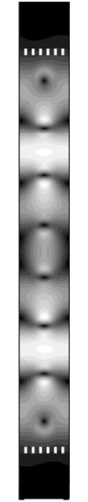

(e)
FIG. 8. Geometry of the slab of PC with $d / a=0.4, \varepsilon_{r}=11.4$, and $N=8$ within a grating period $a_{x}=a$ [plot (a)], and the amplitude of electric field at $k a=2.99$ and $\theta=35^{\circ}$ [plot (b)], $k a=2.949$ and $\theta$ $=37.5^{\circ}$ [plot (c)], $k a=2.923$ and $\theta=40^{\circ}$ [plot (d)], and $k a=3.009$ and $\theta=42^{\circ}$ [plot (e)]; plots (c) and (d) correspond to the same mountain of $T=1$ as that shown in Fig. 7 by asterisk; the brighter the tone, the larger the amplitude is in each of plots (b)-(e); dashed white lines show virtual boundaries of the slab of PC at $y=0$ and $y=N a$.

logs of those in the classical Fabry-Pérot resonators, where the space between the mirrors (interfaces) is either a homogeneous medium or simply air. In other words, the slab of PC can partially mimick near- and far-field effects, which are typical for the Fabry-Pérot resonators, rather than be entirely equivalent to such a resonator. This example also shows that localization can be much stronger in the conventional dielectic PCs with the left handedness than in the slabs of a homogeneous negative-index medium. ${ }^{17}$

\section{E. Increasing $Q$}

As has been demonstrated in Table I, $Q$ factor that exceeds $10^{4}$ can be obtained for the peaks of $T$, which appear within the minipassband. However, much larger values can be achieved by using the same mechanism. Figure 9 presents an example, in which $Q \approx 1.5 \times 10^{6}$. Here, the observed peak of $T$ is the second lowest peak of the minipassband. In turn, for a thinner slab with $N=28, Q \approx 4.5 \times 10^{5}$ for the second lowest peak, which is shifted now to $k a=2.9445$ because of

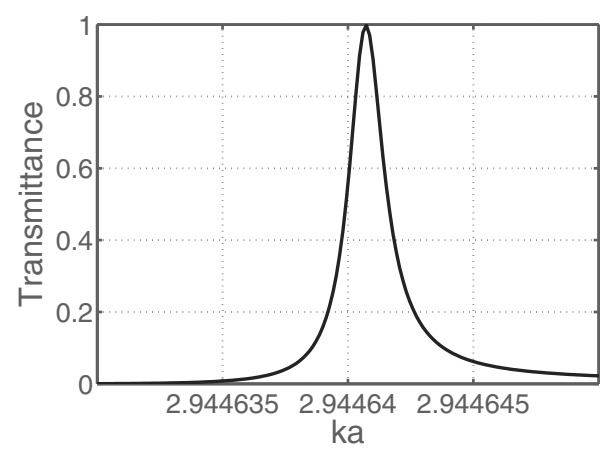

FIG. 9. Transmittance for a narrow range of $k a$ variation at $\theta$ $=37.5^{\circ}, N=36$, and same remaining parameters as in Fig. 5 . 
the variation in $N$ while $\theta$ is fixed. Furthermore, even more advantageous designs, in which $Q>10^{6}$, are possible for thinner slabs of PC owing to a proper choice of $\varepsilon_{r}$ and $d / a$. It follows from the obtained results that $n_{T}>80$ can be obtained at $\theta=37.5^{\circ}$ from the locations of the first and second lowest near-edge peaks of $T$ by taking $N \geq 28$. Such large values of $n_{T}$ are achievable due to the peculiar location of the mountains and valleys of $T$ with respect to each other, which occurs inside the range bounded by dotted lines in Fig. 7.

\section{CONCLUSIONS}

To summarize, defect-mode-like transmission can appear within a narrow, higher-order passband of a PC without defects, leading to similar far- and near-field effects as those being typical for PCs with defects. In particular, multiple, narrow, well-isolated transmission peaks and large values of the equivalent group index of refraction can be obtained at a proper adjustment of the PC parameters. In the considered examples, the largest values of $Q$ factor correspond to the case when a negatively refracted beam is one of the two beams, or a sole beam contributing to the transmission through the slab of PC, while the left handedness takes place.
Large values of $Q$ can also be obtained for a positively refracted beam. The observed effects may be associated with large reflectance of mirrors of an equivalent Fabry-Pérot resonator. Our results demonstrate a route of obtaining of slow light, which can be much more flexible regarding the beam width than that based on defect modes. Existence of the desired narrow passband can be easily predicted by using the conventional approach, which is based on the IFCs and wave vector diagrams. The obtained results show that the defect-free PCs can be an alternative to the PCs with defects for obtaining the operation regimes, which can be utilized in multifrequency filters, concentrators, buffers, and other optical and microwave devices.

\section{ACKNOWLEDGMENTS}

This work is supported by the European Union under the projects PHOME, ECONAM, N4E, and TUBITAK under Projects No. 109E301, No. 107A004, and No. 107A012, and DPT under the project DPT-HAMIT. A.E.S. was supported by the Deutsche Forschungsgemeinschaft under Project No. SE1409.2-1. E.O. acknowledges partial support from the Turkish Academy of Sciences.

*serebryannikov@tu-harburg.de

${ }^{1}$ Photonic Crystals: Physics, Fabrication and Applications, edited by K. Inoue and K. Othaka (Springer, Berlin, 2004).

${ }^{2}$ T. Ueda, K. Othaka, N. Kawai, and K. Sakoda, J. Appl. Phys. 84, 6299 (1998)

${ }^{3}$ H. Benisty, C. Weisbuch, D. Labilloy, M. Rattier, C. J. M. Smith, T. F. Krauss, R. M. De La Rue, R. Houdre, U. Oesterle, C. Jouanin, and D. Cassagne, J. Lightwave Technol. 17, 2063 (1999).

${ }^{4}$ E. Özbay, G. Tuttle, J. S. McCalmont, R. Biswas, M. Sigalas, C. M. Soukoulis, and K. M. Ho, Appl. Phys. Lett. 67, 1969 (1995).

${ }^{5}$ A. Talneau, Ph. Lalanne, M. Agio, and C. M. Soukoulis, Opt. Lett. 27, 1522 (2002).

${ }^{6}$ A. Sugitatsu, T. Asano, and S. Noda, Appl. Phys. Lett. 84, 5395 (2004).

${ }^{7}$ J. Zimmermann, M. Kamp, A. Forchel, and R. Maerz, Opt. Commun. 230, 387 (2004).

${ }^{8}$ M. Koshiba, J. Lightwave Technol. 19, 1970 (2001).

${ }^{9}$ H. Takano, Y. Akahane, T. Asano, and S. Noda, Appl. Phys. Lett. 84, 2226 (2004).

${ }^{10}$ S. Kim, I. Park, and H. Lim, Opt. Lett. 30, 257 (2005).

${ }^{11}$ B. Temelkuran, E. Ozbay, J. P. Kavanaugh, G. Tuttle, and K. M. Ho, Appl. Phys. Lett. 72, 2376 (1998).

${ }^{12}$ J. B. Khurgin, J. Opt. Soc. Am. B 22, 1062 (2005).

${ }^{13}$ J. H. Wu, L. K. Ang, A. Q. Liu, H. G. Teo, and C. Lu, J. Opt. Soc. Am. B 22, 1770 (2005).

${ }^{14}$ H.-Y. Lee, S.-J. Cho, G.-Y. Nam, W.-H. Lee, T. Baba, H. Makino, M.-W. Cho, and T. Yao, J. Appl. Phys. 97, 103111 (2005).

${ }^{15}$ M. Bayindir, B. Temelkuran, and E. Ozbay, Phys. Rev. Lett. 84, 2140 (2000).

${ }^{16}$ M. Makhan and S. K. Ramchurn, J. Opt. Soc. Am. B 24, 3040 (2007).

${ }^{17}$ T. J. Cui, Q. Cheng, W. B. Lu, Q. Jiang, and J. A. Kong, Phys. Rev. B 71, 045114 (2005).

${ }^{18}$ C. Luo, S. G. Johnson, J. D. Joannopoulos, and J. B. Pendry, Phys. Rev. B 65, 201104 (2002).

${ }^{19}$ I. Bulu, H. Caglayan, and E. Ozbay, Appl. Phys. Lett. 83, 3263 (2003).

${ }^{20}$ A. E. Serebryannikov, A. Y. Petrov, and E. Ozbay, Appl. Phys. Lett. 94, 181101 (2009).

${ }^{21}$ K. Sakoda, Optical Properties of Photonic Crystals (Springer, Berlin, 2001), pp. 92-93.

${ }^{22}$ W. Zhong and X. Zhang, Phys. Rev. A 81, 013805 (2010).

${ }^{23}$ See www.cst.com.

${ }^{24}$ T. Magath and A. E. Serebryannikov, J. Opt. Soc. Am. A 22, 2405 (2005).

${ }^{25}$ M. Born and E. Wolf, Priciples of Optics: Electromagnetic Theory of Propagation, Interference and Diffraction of Light (Pergamon Press, Oxford, 1970), pp. 325-326.

${ }^{26}$ T. Baba, Nat. Photonics 2, 465 (2008).

${ }^{27}$ J. B. Pendry, A. J. Holden, W. J. Stewart, and I. Youngs, Phys. Rev. Lett. 76, 4773 (1996).

${ }^{28}$ B. Gralak, S. Enoch, and G. Tayeb, J. Opt. Soc. Am. A 17, 1012 (2000).

${ }^{29}$ S. Foteinopoulou and C. M. Soukoulis, Phys. Rev. B 72, 165112 (2005).

${ }^{30}$ A. E. Serebryannikov, Phys. Rev. B 80, 155117 (2009). 